\title{
Regulation of negative emotions in high trait anxious individuals: an ERP study
}

\author{
Izabela Mocaiber ${ }^{1}$, Mirtes Garcia Pereira ${ }^{2}$, Fátima Smith Erthal ${ }^{2}$, Ivan Figueira ${ }^{1}$, Walter Machado- \\ Pinheiro ${ }^{2}$, Maurício Cagy ${ }^{2}$, Eliane Volchan ${ }^{1}$ and Letícia de Oliveira ${ }^{2}$ \\ 1 Universidade Federal do Rio de Janeiro, Brazil \\ 2 Universidade Federal Fluminense, Brazil
}

\begin{abstract}
Literature has shown that failures in the ability to down-regulate negative emotions are the core substrate of anxiety disorders. Previous studies have investigated this issue by encouraging individuals to voluntarily change how they think about a situation in order to decrease its emotional impact. The majority of studies has demonstrated that explicit instructions to reduce negative affect in anxious individuals are usually ineffective. Thus, the goal of the present study was to investigate whether an implicit regulation strategy would modulate electrophysiological activity (Late Positive Potential) associated to affective picture viewing. The Late Positive Potential (LPP) is a sustained positive deflection in the event-related potential that is larger following the presentation of emotional compared to neutral visual stimuli. Participants (low trait anxious - LTA and high trait anxious - HTA individuals) performed an attention task (bar orientation discrimination) while emotional distractive pictures were presented. The task was performed in two different contexts: in the Real context, participants were informed that the distractive pictures had been obtained from real life situations, whereas in the Fictitious context they were told that the pictures had been obtained from movie scenes. In this vein, we encouraged participants to change how they appraised the pictures. Results showed that HTA individuals exhibited larger Late Positive Potential (LPPs) when mutilation pictures were presented in the Real context. Importantly, during the Fictitious one (regulation strategy) the LPP was reduced even in HTA individuals, emphasizing its importance to psychotherapeutic interventions. The present results indicate that HTA individuals are susceptible to modifications in affective contexts. Keywords: Late Positive Potential, emotion regulation, anxiety, reaction time, unpleasant pictures.
\end{abstract}

Received 22 July 2009; received in revised form 8 October 2009; accepted 22 October 2009. Available on line 29 December 2009.

\section{Introduction}

The disrupting effects of threatening material in the allocation of attention represents one of the strongest neuropsychological correlates of anxiety disorders (Fox, Russo, Bowles \& Dutton, 2001; Koster, Crombez, Verscuere, Van \& Wiersema, 2006; Mogg \& Bradley, 1998; Williams, Mathews \& MacLeod, 1996), reflecting an attention bias to threat. Several authors have suggested that the attentional system of anxious individuals may be abnormally sensitive to threat-related stimuli, leading to a

Izabela Mocaiber and Fátima Smith Erthal, Universidade Federal do Rio de Janeiro, Brazil. Mirtes Garcia Pereira, Walter Machado-Pinheiro, Maurício Cagy and Letícia de Oliveira, Universidade Federal Fluminense, Brazil. Ivan Figueira, Instituto de Psiquiatria, Universidade Federal do Rio de Janeiro, Brazil. Eliane Volchan Institute of Biophysics Carlos Chagas Filho, Universidade Federal do Rio de Janeiro, Brazil. Correspondence regarding this article should be directed to: Letícia de Oliveira, Biomedical Institute, Universidade Federal Fluminense, Rua Professor Hernani Melo, 101, São Domingos, Niterói, Rio de Janeiro, Brazil, CEP 24210-130. Phone: +55-21-9182-0177. E-mail: ldol@biof.ufrj.br pronounced processing bias in favor of threat as compared to non-anxious individuals (Bar-Haim et al., 2007; Goldin, Manber, Hakimi, Canli \& Gross, 2009; Mogg \& Bradley, 1998). Anxiety has also been associated with abnormal processing of safety signals (Kashdan, 2007). Additionally, previous studies have highlighted that failures in the ability to down-regulate negative emotions are the core substrate of anxiety disorders (Cloitre, Koenen, Cohen \& Han, 2002; Mennin, Heimberg, Turk \& Fresco, 2005). In fact, literature has shown that anxious individuals frequently use maladaptive emotion regulation strategies, such as suppression (Campbell-Sills et al., 2006). Suppression involves modifying an emotional response once it is already activated. During suppression, individuals can inhibit the outward expression of emotion (e.g. facial expression), but do not alleviate subjective experience of emotion (CampbellSills, Barlow, Brown \& Hofmann, 2006).

Previous studies, in non clinical samples, have demonstrated that affective processing depends on several factors, including attention and cognitive modulation (Erthal et al., 2005; Ochsner et al., 2004; Ochsner \& Gross, 2005; Oliveira et al., 2009; Pessoa, Padmala \& Morland, 2005). For example, manipulation of the focus of spatial 
attention has been shown to reduce, or even eliminate, differential signals evoked by fearful faces in both functional magnetic resonance imaging (fMRI) and eventrelated potential (ERP) studies (Blair, et al., 2007; Pessoa et al., 2002; Pessoa, McKenna, Gutierrez \& Ungerleider, 2005; Raymond, Fenske \& Tavassoli, 2003). Furthermore, cognitively changing the meaning of emotional stimuli affects evoked responses in the amygdala and other brain areas (Ochsner et al., 2004; Ochsner \& Gross, 2005). More recently, researchers have begun to examine how instructions to alter a participant's reaction to emotional stimuli affect physiology and brain activity (Moser, Hajcak, Bukay \& Simons, 2006; Ochsner et al., 2004; Phan et al., 2005). Results from fMRI studies suggest that instructions to diminish responses to unpleasant images results in decreased amygdala activity, whereas instructions to enhance responses results in increased amygdala activity (Ochsner et al., 2004; Phan et al., 2005).

The impact of cognitive modulation on affective processing has also been addressed in studies using ERPs. The excellent temporal resolution of ERPs has allowed for greater insight into the time course of emotion regulation processes, and these studies suggest that reappraisal modulates ERPs following unpleasant pictures (Foti \& Hajcak, 2008; Moser et al., 2006). These studies focused on the Late Positive Potential (LPP) in particular, which has been showing to be reliably increased in magnitude following both pleasant and unpleasant compared to neutral pictures (Schupp et al., 2000). Thus, the LPP corresponds to a sustained positive deflection in the event-related potential that is larger following the presentation of emotional visual stimuli. Moser and colleagues (2006), for example, found a significant reduction of LPP during the reevaluation of emotional responses. In fact, LPP has been used to index augmented attention to arousing stimuli and results have shown that emotional pictures elicit a larger LPP than neutral ones (Schupp et al., 2000; Hajcak, Moser \& Simons, 2006).

Here, we investigated whether the emotional impact of unpleasant stimuli on electrophysiological activity could be reduced, especially in high trait anxious (HTA) individuals, by two different emotional regulation strategies. To accomplish that, the first strategy adopted was "attentional deployment", which consisted of diverting attention away from emotional stimuli (Ochsner \& Gross, 2005). According to previous studies, attentional deployment can effectively reduce differential responding to emotional items (Erthal et al., 2005; Pessoa et al., 2002; Pessoa et al., 2005). In this vein, emotional stimuli were presented as distractors (out of attentional focus) during the execution of a relevant attention task. This approach seems particularly interesting because it simulates a naturalistic situation, in which multiple stimuli dynamically compete for attention in the environment. The second strategy consisted of an "implicit reappraisal strategy" that promoted a cognitive re-evaluation of stimuli (Foti \& Hajcak, 2008; Oliveira et al., 2009). Specifically, subjects viewed neutral and unpleasant pictures during the execution of an attentional task in two different contexts. In the Fictitious context, they read a text that informed that the unpleasant pictures were taken from movie production, whereas in the Real context, they read that the pictures corresponded to day-life scenes. We reasoned that electrophysiological responses evoked during the fictitious condition would be attenuated relative to the condition in which the pictures were described as real, especially because the mutilation pictures were presented as distracters. As already pointed previously, affective processing can be abolished in conditions of few attentional resources available.

In summary, we hypothesized that emotional responses, indexed by reaction time and magnitude of event-related potentials (LPPs), would be modulated by the combination of two emotional regulation strategies: "attentional deployment" and "implicit reappraisal". We expected that the magnitude of the LPP, an index of attention and affective processing, would be augmented during the viewing of unpleasant pictures, in the Real context. Furthermore, we expected that during the Fictitious context, where both strategies were present, the LPP would be reduced. We were especially interested in investigating this mechanism in HTA individuals, in order to investigate their ability to down-regulate negative emotion.

\section{Methods}

\section{Participants}

Thirty right-handed healthy undergraduate students (15 male, mean age of 21.30 years, $\mathrm{SD}=1.79$ ) participated in this study. The electrophysiological recordings were obtained from 21 participants (12 male, mean age of 21.31 years, $\mathrm{SD}=1.81$ ). Volunteers were selected among students from the Federal Fluminense University, and had normal or corrected-to-normal vision. They reported no psychiatric or neurologic problems and were not under medication acting in the nervous system. Subjects were naive as to the purpose of the experiment. Before the data collection, all procedures were approved by the local ethics committee and subjects gave informed consent to participate.

\section{Stimuli}

All images used in the experiment (72 neutral and 72 unpleasant) were taken from the International Affective Picture System (IAPS) (Lang, Bradley \& Cuthbert, 1997) and from the World Wide Web. Following the protocol developed by Lang and colleagues, all images were assessed on a 1-9 scale in terms of valence (from negative to positive) and arousal (from low to high) by a separate group of graduate students $(\mathrm{N}=20)$ with ages similar to the current subjects $(22.3, \mathrm{SD}=1.8)$. 
Unpleasant and neutral images differed significantly from each other in IAPS normative ratings of valence $(M=2.08$ and 5.21, respectively, $\mathrm{t}=-58.02, \mathrm{p}<.001)$ and arousal $(M=6.6$ and 3.4, respectively, $\mathrm{t}=34.43$, $\mathrm{p}$ $<.001)$. We randomized the presentation of each picture across the different contexts to avoid differences in valence and arousal among contexts. Each picture was presented twice and this repetition occurred only inside the sub-blocks of the same context.

The experiment was conducted in a sound-attenuated room under indirect lighting. Subjects sat in front of a computer monitor. The distance from the subject's eye to the display was $57 \mathrm{~cm}$ (the position was ensured by means of a chinrest). Stimuli were presented using the software Presentation (Neurobehavioral Systems, Inc.; Albany, CA).

\section{Procedure}

Participants were tested individually. On arrival, subjects completed the Portuguese trait section (Gorenstein \& Andrade, 1997) of the State and Trait Anxiety Inventory (STAI-T) (Spielberger, 1983). This questionnaire consists of 20 short statements (e.g. "I feel afraid") rated on a four-point scale ( 1 = seldom/ never, $4=$ very often/always). This procedure was adopted to separate the sample in low and high trait anxious individuals.

After a brief description of the experiment, electroencephalograph sensors were attached and the subject was given detailed task instructions. All participants performed a practice session to assure that they understood the task. Then, the experimental session started. Each trial was initiated with a fixation cross, shown for 500 to 900 ms. Then, a central picture $\left(9^{\circ} \times 12^{\circ}\right)$ and two peripheral bars $\left(0.3^{\circ} \times 3.0^{\circ}\right)$ were simultaneously shown for $200 \mathrm{~ms}$. The bars were presented at $9^{\circ}$ to the right and left of the center of the picture (Figure 1). A whole-screen gray scale checkerboard mask was then shown, and it remained on the screen until the subject responded to the peripheral bars by pressing a button. Volunteers were instructed to ignore the central images irrelevant to the task and to respond as quickly and as accurately as possible, indicating whether or not the orientations of the peripheral bars were the same. Two classes of images were employed as distractors: neutral and unpleasant. Neutral pictures consisted of photographs of people in "normal life" situations and unpleasant images consisted of photographs of mutilated bodies. The angular difference of the bars was $90^{\circ}$ or $6^{\circ}$ on non-match trials. Buttons (right or left index finger) corresponding to same (match)/different (non-match) orientation were counterbalanced across subjects. Each block contained an equal number of match and non-match trials.

Subjects participated in one experimental session that comprised two counterbalanced contexts (fictitious and real). The contexts contained two blocks divided in 4 sub-blocks. Each sub-block included 18 trials, where unpleasant and neutral pictures were randomly presented

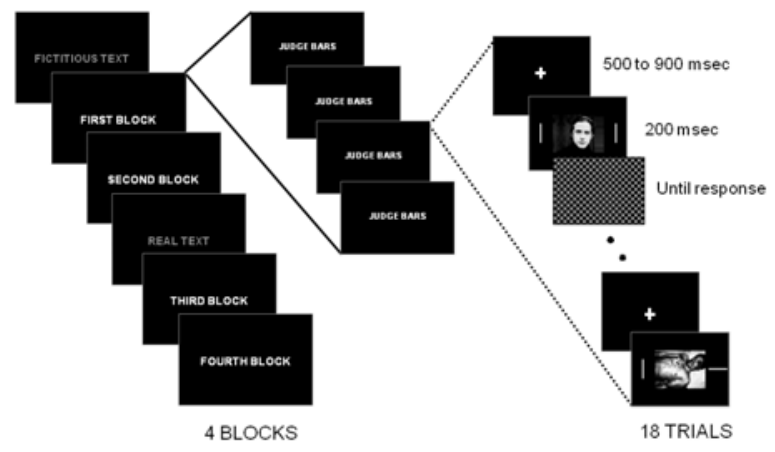

Figure 1. Experimental design. A central fixation cross was presented for 500 to $900 \mathrm{~ms}$, followed by a central picture and two peripheral bars, presented simultaneously (for $200 \mathrm{~ms}$ ) to the right and left of the fixation point. Then a checkerboard-like mask was presented remaining on the screen until a response was given or 1,500 ms had elapsed. Subjects were instructed to ignore the central picture and attend to the peripheral bars, responding with a finger press as quickly and accurately as possible as to whether the bars were in the same or in a different orientation.

in the same proportion. The attentional task instruction was presented in the beginning of each sub-block. The time interval between blocks was approximately 3 minutes.

In the beginning of each experimental context, subjects read a text containing technical information about the origins of the distractive pictures. In one condition, they read that the unpleasant pictures were fictitious whereas in the other, they read that the pictures corresponded to real scenes. This information was given in a very subtle way, in order to reduce subject's awareness about the experimental manipulation. Thus, participants performed the bar-orientation task under different contexts that described the pictures as "fictitious" or "real".

For the fictitious condition, participants read the following text: "The pictures that will be shown to you in the next trials were obtained from movies with the aim of convincing the audience. Therefore, the pictures were produced by means of diverse techniques such as makeup, and do not correspond to real situations". For the real context, participants read the following text: "The IAPS is a set of standardized colored photographs of a wide range of daily-life situations. All the pictures are real and were obtained from the web, media, or taken by the group that developed the IAPS". It is important to note that the pictures that were presented to some volunteers in the Real context were presented in the Fictitious one to others, in a randomized fashion. This procedure guaranteed that any attenuation effect would not be associated to a specific pool of pictures.

\section{Behavioral Analysis}

A 2 (context: fictitious and real) x 2 (stimulus valence: neutral and unpleasant) x 2 (anxiety trait: low trait anxious - LTA and high trait anxious - HTA) ANOVA was conducted on the reaction time (RT) 
data. All variables were within-subjects, except for anxiety trait. Follow-up analyses were used for specific comparisons $(\alpha=.05)$. For this ANOVA, the total sample $(\mathrm{N}=30)$ was separated into high (HTA) and low trait anxiety (LTA) groups using a mean split on STAItrait scores. The LTA group consisted of 18 individuals (9 male, mean age $=21.33$ years, mean STAI-T score $=31.72, \mathrm{SD}=5.09)$ and the HTA group comprised 12 individuals ( 5 male, mean age $=21.25$ years, mean STAI-T score $=48.16, \mathrm{SD}=5.84)$.

\section{Event Related Potential Recording and Data Analysis}

EEG was acquired from three scalp sites (Fz, Cz and $\mathrm{Pz}$, according to the 10-20 International System), referenced to the left earlobe and grounded at FPz, using silvertip electrodes (for details, see Fabiani, Graton \& Coles, 2000). Signal was conditioned and digitized at the rate of $240 \mathrm{~Hz}$ using the BIOPAC System (BIOPAC Systems, Inc., USA). The signal was further filtered via a high-pass filter $(.05 \mathrm{~Hz})$, a low-pass filter $(30 \mathrm{~Hz})$, and notch filtered $(60 \mathrm{~Hz})$ to remove main-line interference. Epochs were extracted from raw EEG and contained signals between 100 ms before and $1000 \mathrm{~ms}$ after trial onset. Artifact removal was performed automatically by rejecting epochs with signal changes exceeding $100 \mu \mathrm{V}$. Baseline correction considered the average voltage of $100 \mathrm{~ms}$ as the prestimulus interval. Because the early window of the LPP is maximally expressed at more posterior scalp locations (Foti \& Hacjak, 2008; Hajcak \& Nieuwenhuis, 2006; Olofsson, Nordin, Sequeira \& Polich, 2008) we conducted an ANOVA that focused on $\mathrm{Cz}$ and $\mathrm{Pz}$ electrodes, as a cluster. Specifically, ERPs were constructed by averaging trials as a function of the following factors: 2 (anxiety trait: LTA and HTA) x 2 (context: fictitious and real) x 2 (stimulus valence: neutral and unpleasant). The ERP component was defined as the largest positive-going peak that occurred within the 300-600 ms window following trial onset (initial phase of the Late Positive Potential - LPP). All variables were within-subjects, except for trait anxiety.

For this ANOVA, the total sample $(\mathrm{N}=21)$ was separated into HTA and LTA groups. The LTA group consisted of 12 individuals ( 7 male, mean age $=21.50$ years, mean STAI-T score $=30.25, \mathrm{SD}=5.04$ ) and the HTA group comprised 9 individuals (2 male, mean age $=21$ years, mean STAI-T score $=50.44, \mathrm{SD}=4.74$ ) . In fact, we wanted to investigate whether the general pattern of emotional reactivity and regulation observed in the present sample would differ as a function of individual predispositions (Heim \& Nemeroff, 2001; Pole et al., 2007; Souza et al., 2007). The LPP amplitude was statistically evaluated using the software Statistica (version 6.0) with Greenhouse-Geisser corrections when necessary. The Newman-Keuls procedure was used for all post-hoc comparisons $(\alpha=.05)$.

\section{Results}

\section{Behavioral Performance}

The 2 (context $\mathrm{x} 2$ valence $\mathrm{x} 2$ trait anxiety) mixeddesign ANOVA did not reveal a three-way interaction $(\mathrm{F}=1.58, \mathrm{df}=1,28, \mathrm{p}=.21)$. Thus, reaction time within each emotional context was not modulated by anxiety trait. There was a tendency for a CONTEXT $\mathrm{x}$ VALENCE interaction $(\mathrm{F}=3.98, \mathrm{df}=1.28, \mathrm{p}=.06)$. A follow-up analysis showed that in the real context, RT tended to be slower during the viewing of unpleasant (mean $=609.1 \mathrm{~ms}, \mathrm{SD}=131.8$ ) relative to neutral pictures (mean $=597.7 \mathrm{~ms}, \mathrm{SD}=119.7 ; \mathrm{p}=.08$ ). In the fictitious condition, this slowing down was not observed $(p=.42)$.

\section{Event related potential}

Because the early window of the LPP is maximally expressed at more posterior scalp locations, we conducted an ANOVA that focused on $\mathrm{Cz} / \mathrm{Pz}$ electrodes. Additionally, the Fz electrode signal had excessive artifact.

The 2 (context) x 2 (valence) x 2 (anxiety trait) mixed-design ANOVA on the LPP values obtained for the $\mathrm{Cz} / \mathrm{Pz}$ cluster revealed a triple interaction $(\mathrm{F}=5.37$, $\mathrm{df}=1.19, \mathrm{p}=.04)$. Newman-Keuls post hoc analysis showed that LPP amplitude during the viewing of mutilation pictures was significantly larger in the Real condition relative to the Fictitious one in the HTA (mean $=5.78$ and $4.36 \mu \mathrm{V}, \mathrm{SD}=3.05$ and 3.38, respectively) $(\mathrm{p}<.001)$ and LTA group (mean $=8.52$ and $7.52 \mu \mathrm{V}$, $\mathrm{SD}=3.30$ and 3.81, respectively) $(\mathrm{p}<.001)$. LPP amplitudes during viewing of negative images was significantly larger in comparison to neutral ones in the real condition for the HTA group only, indicating an attentional bias in individuals with high anxiety scores ( $\mathrm{F}=8.52$ and $7.54 \mu \mathrm{V}, \mathrm{SD}=3.30$ and 3.54 , respectively, $\mathrm{p}<.01)$. Interestingly, this difference was not observed in the fictitious condition ( $\mathrm{F}=7.52$ and $7.90 \mu \mathrm{V}, \mathrm{SD}$ $=3.81$ and 4.08 , respectively, $\mathrm{p}=.28$ ), indicating that the HTA group had a pronounced emotional reactivity to mutilation images of the real condition and was able to regulate this response in the fictitious condition. Figure 2 shows the mean amplitude values of the LPP for all subjects and as a function of anxiety scores.

\section{Discussion}

In the present study, we observed that high trait anxious (HTA) individuals were able to diminish the emotional impact of unpleasant stimuli (indexed by electrophysiological activity) by means of two combined emotion regulation strategies (attentional deployment and implicit reappraisal). During the real context, unpleasant task-irrelevant stimuli elicited larger responses (assessed by the Late Positive Potential) than neutral ones. This emotional enhancement of the LPP was specifically observed in 
HTA individuals. Importantly, there was an attenuation of LPP amplitudes to unpleasant pictures during the fictitious context, in comparison to the Real one, even to HTA individuals.

Our data showed that the magnitude of the LPP was reliably reduced following a more neutral description, indicating that changes in narrative are sufficient to modulate electrocortical responses. Taken together, these data demonstrate that cognitive strategies such as reappraisal reduce the intensity of negative experience and brain activity.

Our results corroborate previous studies that showed an augmentation of the LPP in response to unpleasant and pleasant pictures (Foti \& Hajcak, 2008; Hajcak \& Nieuwenhuis, 2006; Moser et al., 2006). In a study by Hajcak and colleagues (2006) ERP activity was sensitive to the way stimuli were judged. Subjects viewed both pleasant and unpleasant pictures and categorized them along an affective or non-affective dimension. They found that the Late Positive Potential was augmented for both pleasant and unpleasant pictures when participants made affective, compared with nonaffective judgments. This enhancement of the LPP has been interpreted as an index of attention and orienting processes (Schupp et al., 2000).

In the fictitious context, emotional responses were attenuated, indicating that cognitive modulation was efficient to reduce the impact of negative stimuli. In this experimental condition, subjects read an instruction that informed that the pictures were not real. Thus, subjects were encouraged, in a subtle way, to use an implicit reappraisal strategy. The use of different descriptions previous to picture presentation aiming to modulate its emotional impact has also been used in a recent paper by Foti \& Hajcak, (2008). The authors examined whether a more or less negative description preceding the presentation of unpleasant images would modulate the LPP. This component of the ERP was recorded from subjects while they viewed unpleasant and neutral images and heard a brief description of the upcoming picture. Results showed that the magnitude of the LPP was reliably reduced following a more neutral description, indicating that changes in narrative are sufficient to modulate electrocortical responses. Taken together, these data demonstrate that cognitive strategies such as reappraisal reduce the intensity of negative experience and brain activity.

Regarding the anxiety traits, we observed that LPP amplitudes during the viewing of unpleasant pictures in the real context were larger in comparison to neutral ones in the HTA group only. This result is in accordance with the attentional bias to negative information already described for anxious individuals (Koster et al., 2006; Mogg \& Bradley, 1998; Williams et al., 1996). Koster and colleagues (2006), for example, investigated whether the attentional bias in high trait anxious individuals would

Figure 2. Mean Late Positive Potential (collapsed across channels) amplitudes as a function of context, valence and anxiety traits. LPP amplitude during viewing of mutilation pictures was significantly larger in the Real condition relative to the Fictitious one in the HTA and LTA group $(\mathrm{p}<.001)$. During viewing of negative images, LPP was significantly larger in comparison to neutral ones in the Real condition for the HTA group only $(\mathrm{p}<.01)$. This difference was not observed in the fictitious condition $(\mathrm{p}=.28) . \mu \mathrm{V}=$ microvolts, $\mathrm{LPP}=$ Late Positive Potential, $\mathrm{LTA}=$ low trait anxious, HTA = high trait anxious

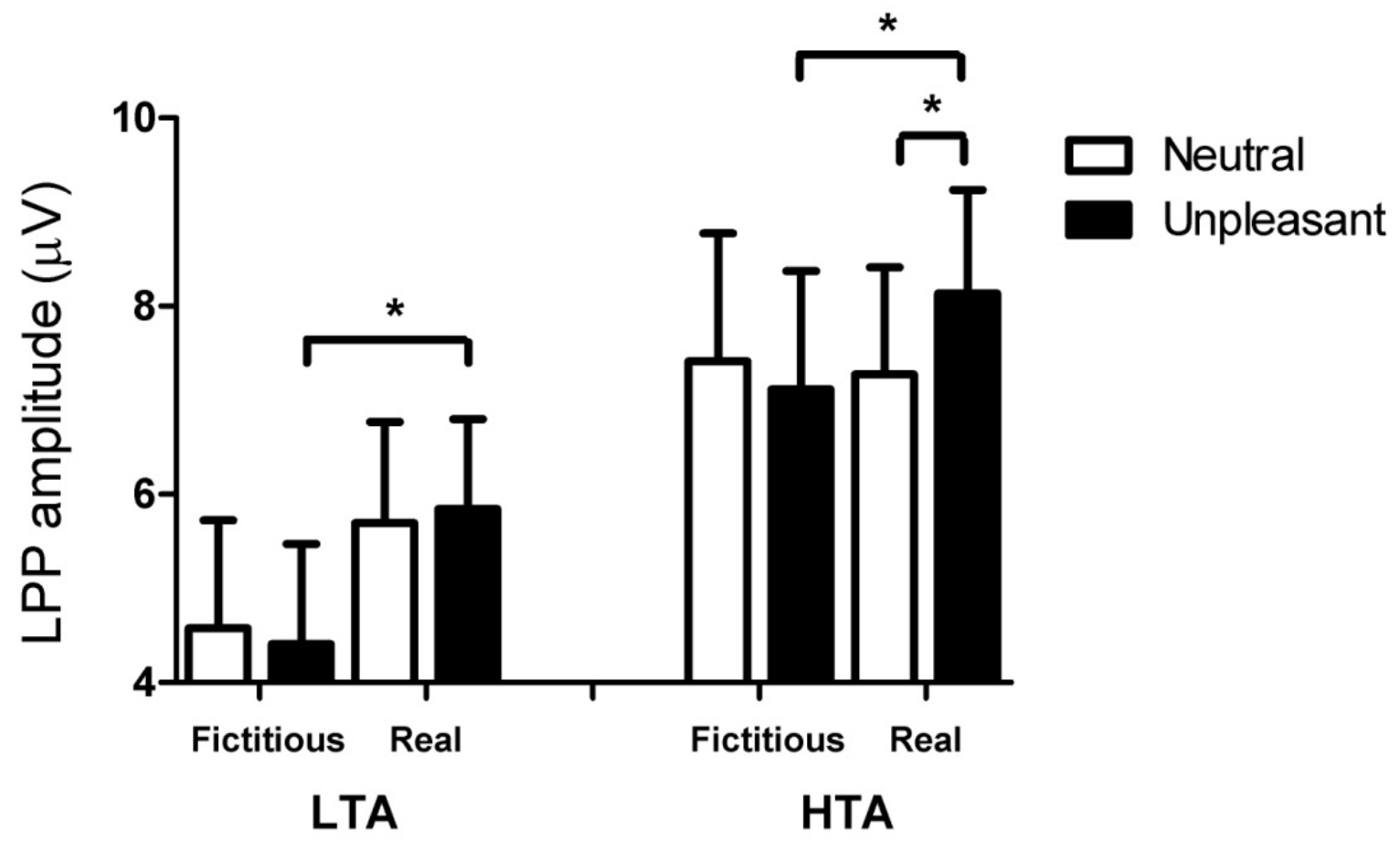


be related to facilitated attentional engagement to threat or difficulties in disengaging attention from threat. LTA and HTA individuals performed an exogenous cueing test, in which the location of a target was correctly or incorrectly cued by neutral, highly and mildly threatening pictures. Their results revealed that at 100 ms picture presentation, HTA individuals more strongly engaged their attention with and showed impaired disengagement from highly threatening pictures than LTA individuals. These findings emphasize the role of individual predispositions and traits on emotional responding (Hariri et al., 2002; Souza et al., 2007)

One limitation of the present study was that the number of male and female in the HTA and LTA groups was not balanced. It is possible that the increased reactivity during the Real context, observed only for the HTA individuals, may be due to the fact that this group was composed mainly by women. Indeed, Bradley and colleagues showed that women exhibited a broad disposition to respond with greater defensive reactivity to aversive pictures (Bradley, Codispoti, Sabatinelli \& Lang, 2001). Moreover, epidemiological studies indicate that women are at a higher risk for affective disorders, such as anxiety and depression, suggesting a higher incidence of negative affect (Sachs-Ericsson \& Ciarlo, 2000). On the other hand, other studies have shown that women exhibit a pattern of behavior and physiological response similar to that observed in men when exposed to the same emotional stimuli (Kring \& Gordon, 1998; Pereira et al., 2004). As suggested by Pereira and colleagues (2004), one possibility is that women and men differ only in the expression of emotion, but that the responses and influences of emotional stimulation are the same in both sexes. Along this vein, Gross and John (1998) pointed out that men mask their feelings more than do women, which suggests an expressive difference, and not necessarily an experiential difference, between men and women.

Interestingly, in the present study, during the fictitious condition, the emotional modulation was not observed, indicating that although HTA individuals reacted more, they were able to take advantage of the regulation strategies. Concerning the implicit reappraisal strategy, a number of studies have shown that re-evaluating an emotional situation can change the nature of its meaning, leading to the attenuation of its relevance (Eipert et al., 2007; Goldin, McRae, Ramel \& Gross, 2008; Moser et al., 2006; Ochsner et al., 2004; Phan et al., 2005). The present study showed a significant attenuation of emotional responses at electrophysiological level by using a combination of two emotion regulation approaches. The modulation was found even in HTA individuals, indicating the effectiveness of joining both strategies. This seems particularly important once it has been shown that anxious individuals exhibit exaggerated emotional reactivity and reduced ability to voluntarily implement emotion regulation (Campbell-Sills et al., 2006; Koster et al., 2006; Williams et al., 1996).

Understanding the beneficial effects of emotion regulation becomes particularly interesting to psychotherapeutic interventions that target anxiety disorders. The notion that reappraisal reduces negative emotional experience is similar to the idea that is the basis for cognitive therapy: that cognition can significantly influence emotion. In fact, therapeutic protocols for psychopathological conditions have incorporated approaches to reestablish the abilities to regulate negative emotions (Berking et al., 2008; Campbell-Sills et al., 2006; Mennin et al., 2005). For instance, anxiety disorder treatments such as Cognitive Behavioral Therapy are primarily based on reappraisal mechanisms (Mocaiber et al., 2008; Porto et al., 2009). Actually, deficits in emotion regulation skills have been described not only in anxiety disorders, but seem to be critical to the development and maintenance of a wide range of mental disorders (Gross \& Muñoz, 1995). Thus, one interesting route could be the association of regulation strategies to potentiate the effects of psychotherapeutic interventions. Future studies are necessary to address this issue. In summary, the present findings highlight the importance of targeting emotion-regulation skills in psychological interventions.

\section{Acknowledgments}

This study was supported by CAPES, CNPq, PRONEX/FAPERJ, IBN-net/FINEP, MCT/CNPq, Projeto Institutos do Milênio. We would like to thank Dr. Luiz Pessoa for his comments and important suggestions to this work.

\section{References}

Bar-Haim, Y., Lamy, D., Pergamin, L., Bakermans-Kranenburg, M.J., \& van IJzendoorn, M.H. (2007). Threat-related attentional bias in anxious and nonanxious individuals: a meta-analytic study. Psychological Bulletin, 133, 1-24.

Berking, M., Wupperman, P., Reichardt, A., Pejic, T., Dippel, A., \& Znoj, H. (2008). Emotion-regulation skills as a treatment target in psychotherapy. Behaviour Research and Therapy, 46, 1230-1237.

Blair, K.S., Smith, B.W., Mitchell, D.G., Morton, J., Vythilingam, M., Pessoa, L. et al. (2007). Modulation of emotion by cognition and cognition by emotion. Neuroimage, 35, 430-440.

Bradley, M.M., Codispoti, M., Sabatinelli, D., \& Lang, P.J. (2001). Emotion and motivation II: sex differences in picture processing. Emotion, 1, 300-319.

Campbell-Sills, L., Barlow, D.H., Brown, T.A., \& Hofmann, S.G. (2006). Effects of suppression and acceptance on emotional responses of individuals with anxiety and mood disorders. Behaviour Research and Therapy, 44, 1251-1263.

Cloitre, M., Koenen, K.C., Cohen, L.R., \& Han, H. (2002). Skills training in affective and interpersonal regulation followed by exposure: a phase-based treatment for PTSD related to childhood abuse. Journal of Consulting and Clinical Psychology., 70, 1067-1074.

Eippert, F., Veit, R., Weiskopf, N., Erb, M., Birbaumer, N., \& Anders, S. (2007). Regulation of emotional responses elicited by threat-related stimuli. Human Brain Mapping, 28, 409-423.

Erthal, F.S., Oliveira, L.de, Mocaiber, I., Pereira, M.G., hadoPinheiro, W., Volchan, E. et al. (2005). Load-dependent modulation 
of affective picture processing. Cognitive, Affective, \& Behavioral Neuroscience, 5, 388-395.

Fabiani, M., Graton, G., \& Coles, M.G.H. (2000). Event-related brain potentials: Methods, theory and application. In: Cacioppo, J.T., Tassinary, L.G., \& Berntson, G.G. (Eds.). Handbook of psychophysiology (pp. 53-84). Cambridge, UK: Cambridge University Press.

Foti, D., \& Hajcak, G. (2008). Deconstructing reappraisal: descriptions preceding arousing pictures modulate the subsequent neural response. Journal of Cognitive Neuroscience., 20, 977-988.

Fox, E., Russo, R., Bowles, R., \& Dutton, K. (2001). Do threatening stimuli draw or hold visual attention in subclinical anxiety? Journal of Experimental Psychology: General, 130, 681-700.

Goldin, P.R., Manber, T., Hakimi, S., Canli, T., \& Gross, J.J. (2009). Neural bases of social anxiety disorder: emotional reactivity and cognitive regulation during social and physical threat. Archives of General Psychiatry, 66, 170-180.

Goldin, P.R., McRae, K., Ramel, W., \& Gross, J.J. (2008). The neural bases of emotion regulation: reappraisal and suppression of negative emotion. Biological Psychiatry, 63, 577-586.

Gorenstein, C., \& Andrade, L. (1997). Validation of a Portuguese version of the beck depression inventory and the Sate-Trait Inventory in Brazilian subjects. Brazilian Journal of Medical and Biological Research, 29, 453-457. Gross, J.J., \& John, O.P. (1998). Mapping the domain of expressivity: multimethod evidence for a hierarchical model. Journal of Personality and Social Psychology, 74, 170-191.

Gross, J., \& Muñoz, R. (1995). Emotion regulation and mental health. Clinical Psychology: Science and Practice, 2, 151-164.

Hajcak, G., Moser, J.S., \& Simons, R.F. (2006). Attending to affect: appraisal strategies modulate the electrocortical response to arousing pictures. Emotion, 6, 517-522.

Hajcak, G., \& Nieuwenhuis, S. (2006). Reappraisal modulates the electrocortical response to unpleasant pictures. Cognitive, Affective, \& Behavioral Neuroscience, 6, 291-297.

Hariri, A., Mattay, V., Tessitore, A., Kolachana, B., Fera, F., Goldman, D. et al. (2002). Serotonin transporter genetic variation and the response of the human amygdala. Science, 297, 400-403.

Heim, C., \& Nemeroff, C. (2001). The role of childhood trauma in the neurobiology of mood and anxiety disorders: preclinical and clinical studies. Biological Psychiatry, 49, 1023-1039.

Kashdan, T.B. (2007). Social anxiety spectrum and diminished positive experiences: theoretical synthesis and meta-analysis. Clinical Psychological Review, 27, 348-365.

Koster, E.H., Crombez, G., Verschuere, B., Van, D.S., \& Wiersema, J.R. (2006). Components of attentional bias to threat in high trait anxiety: Facilitated engagement, impaired disengagement, and attentional avoidance. Behaviour Research and Therapy, 44, 1757-1771.

Kring, A.M., \& Gordon, A.H. (1998). Sex differences in emotion: expression, experience, and physiology. Journal of Personality and Social Psychology, 74, 686-703.

Lang, P.J., Bradley, M.M., \& Cuthbert, B.N. (1997). International Affective Picture System (IAPS). Instruction Manual and Affective Ratings. Gainesville, FL, USA, University of Florida, NIMH Center fo the Study of Emotion and Attention.

Mennin, D.S., Heimberg, R.G., Turk, C.L., \& Fresco, D.M. (2005). Preliminary evidence for an emotion dysregulation model of generalized anxiety disorder. Behavior Research and Therapy, 43, 1281-1310.

Mocaiber, I., Oliveira, L., Pereira, M., Machado-Pinheiro, W., Ventura, P., Figueira, I. et al. (2008). Neurobiologia da regulação emocional: implicações para a terapia cognitivo-comportamental. Psicologia em Estudo, 13, 531-538.

Mogg, K., \& Bradley, B.P. (1998). A cognitive-motivational analysis of anxiety. Behaviour Research and Therapy, 36, 809-848.

Moser, J.S., Hajcak, G., Bukay, E., \& Simons, R.F. (2006). Intentional modulation of emotional responding to unpleasant pictures: an ERP study. Psychophysiology, 43, 292-296.

Ochsner, K.N., \& Gross, J.J. (2005). The cognitive control of emotion. Trends in Cognitive Science, 9, 242-249.

Ochsner, K.N., Ray, R.D., Cooper, J.C., Robertson, E.R., Chopra, S., Gabrieli, J.D. et al. (2004). For better or for worse: neural systems supporting the cognitive down- and up-regulation of negative emotion. Neuroimage, 23, 483-499.

Oliveira, L., Oliveira, L., Joffily, M., Pereira Junior, P., Pereira, M., Lang, P. et al. (2009). Autonomic reactions to mutilation pictures: positive affect facilitates safety signal processing. Psychophysiology, $46,870-873$.

Olofsson, J.K., Nordin, S., Sequeira, H., \& Polich, J. (2008). Affective picture processing: an integrative review of ERP findings. Biological Psychology, 77, 247-265.

Pereira, M.G., Volchan, E., Oliveira, L., hado-Pinheiro, W., Rodrigues, J.A., Nepomuceno, F.V. et al. (2004). Behavioral modulation by mutilation pictures in women. Brazilian Journal of Medical and Biological Research, 37, 353-362.

Pessoa, L., McKenna, M., Gutierrez, E., \& Ungerleider, L.G. (2002). Neural processing of emotional faces requires attention. Proceedings of the National Academy of Sciences, U.S.A, 99, 11458-11463.

Pessoa, L., Padmala, S., \& Morland, T. (2005). Fate of unattended fearful faces in the amygdala is determined by both attentional resources and cognitive modulation. Neuroimage, 28, 249-255.

Phan, K.L., Fitzgerald, D.A., Nathan, P.J., Moore, G.J., Uhde, T.W., \& Tancer, M.E. (2005). Neural substrates for voluntary suppression of negative affect: a functional magnetic resonance imaging study. Biological Psychiatry, 57, 210-219.

Pole, N., Neylan, T., Otte, C., Metzler, T., Best, S., Henn-Haase, C. et al. (2007). Associations between childhood trauma and emotionmodulated pscyhophysiological responses to startling sounds: a study of police cadets. J Abnormal Psychology, 116, 352-361.

Porto, P., Oliveira, L., Figueira, I., Volchan, E., Mari, J., \& Ventura, P. (2009). Cognitive Behavioral Therapy change the brain? A Systematic Review of Neuroimage in Anxiety Disorders. Journal of Neuropsychiatry and Clinical Neurosciences, 21, 114-125.

Raymond, J.E., Fenske, M.J., \& Tavassoli, N.T. (2003). Selective attention determines emotional responses to novel visual stimuli. Psychological Science, 14, 537-542.

Sachs-Ericsson, N., \& Ciarlo, J. (2000). Gender, Social roles and mental health: an epidemiological perspective. Sex Roles A Journal Research, 43(9/10), 339-362.

Schupp, H.T., Cuthbert, B.N., Bradley, M.M., Cacioppo, J.T., Ito, T., \& Lang, P.J. (2000). Affective picture processing: the late positive potential is modulated by motivational relevance. Psychophysiology, 37, 257-261.

Souza, G., Mendonça-de-Souza, A., Barros, E., Coutinho, E., Oliveira, L., Mendlowicz, M. et al. (2007). Resilience and vagal tone predict cardiac recovery from acute social stress. Stress, 10, 368-374.

Spielberger C.D. (1983). Manual for the State-trait anxiety inventory. Palo Alto, CA: Consulting Psychologists Press.

Williams, J.M., Mathews, A., \& MacLeod, C. (1996). The emotional Stroop task and psychopathology. PsychologicalBulletin, 120, 3-24. 